\title{
Design of a Cloud-based Print Manufacturing Management System
}

\author{
Yongbin Zhang ${ }^{1}$, Yanping Du ${ }^{1}$, Ronghua Liang ${ }^{2}$ and Shulin Yang ${ }^{3}$ \\ ${ }^{1}$ Department of Mechanical Engineering Beijing Institute of Graphic Communication, China \\ ${ }^{2}$ Department of Network Information Technoloy Beijing Institute of Graphic Communication, China \\ ${ }^{3}$ Department of Information Engineering Beijing Institute of Graphic Communication, China \\ \{zhangyongbin \& duyanping \& liangronghua \& duyanping\}@bigc.edu.cn
}

\begin{abstract}
With the fierce competition and increasing cost of labor, printers face the problem that how to increase their production and efficiency. Information technology is one of the most used ways to achieve that object. Different applications, such as spreadsheet and Enterprise Resource Planning system are used for in different workshops. These applications really help printer improve their production in some extend. After investigating the usage of IT technology in different workshops, we found that most of printers in China have various difficulties when using existing applications. We presented a cloud-based management system as a service to help printers resolve some of the problems found. With cloud-based management system, printers don't need to maintain the running hardware and software system. The system can be accessed from anywhere at any time by authorized people after successfully authenticated. Paper documents are not more needed for communication among departments.

Index Terms - print management, cloud computing,
\end{abstract} manufacturing, software as a service

\section{Introduction}

With the increasing cost of labor and intension of competition, printers nowadays focus more than ever on profits. Over the past ten years, the cost of labor in printing industry has been doubled while the price of printing press for the same requirement is almost the same in China. These factors force printers to think about reducing cost and increasing profits more seriously. More and more printers have realized that Information Technology is an effect way to help them survive.

Spreadsheet, like Microsoft Excel, is widely used as print business management software in most small-median size printing companies [1]. One reason is that spreadsheet is the simplest and least expensive approach to gather information and use basic formulas. The other reason is almost no training required for using spreadsheet. However, printers face many difficulties when using spreadsheet managing their business. Communication among departments is delayed. For examples, when schedule is sent out to all related departments, there is no efficient way to get the status of ongoing tasks besides face-toface or with phone communication. Another more serious problem is that the copy and paste of the content in spreadsheet or between sheets is prone to errors.

Besides spreadsheet, other more powerful and more functions systems, such as enterprise resource planning (ERP) or management information system (MIS) have been adopted in many printing companies. The price for printing ERP ranges from ten thousand Yuan to more than one million Yuan. After investigating the effects of ERP in printing company, we found that ERP systems have improved the capability of printing company information management and solved many problems faced with spreadsheet. However, in most companies we investigated that only department managers can access and use the ERP system. And the manager of a department has to send document printed with ERP system to his employees, which leads the ERP system to low effect.

In this paper, we presented a cloud-based management information system. The system is provided as a service to printer workshops. We deployed the system on cloud computing platform provided by one platform as a service provider. Employees of printing and press companies can access the system from anywhere and use the system after successful authentication. There is no cost for hardware running the system. There is no need for hardware and system maintenance. There system can be accessed from anywhere and anytime as long as the internet connection is available.

\section{Problems Faced By Printers}

There were about 100 thousand printing related companies in China in 2009 according to the statistics. However, more than 95 percent of them had an annual sale less than 5 million Yuan. There were about 7 thousand stateowned companies and more than 40 thousand ones were private [2].

We did a survey on the status about information management in printing company in Beijing in 2012. We chose 4 different printing companies according to the number of employees and annual revenue. We visited 4 private printing companies and talked with the owners and managers face to face. Among them, one had an annual sell more than 100 million Yuan with more than 500 employees. The company bought an ERP system costing 60 thousand Yuan three years ago. Another had about 30 million Yuan with about 200 employees, which had installed an ERP system costing 10 thousand Yuan in 2011. The other two had less than 1 million with about 50 employees and used spreadsheet managing their business.

The two companies with ERP system can input almost all information, such as customers, orders and material information, into their ERP system. However, only few people, such a department manager, general manager can access the 
ERP system. So it is not convenient for monitor the manufacturing process. For example, printing department manager had to print the job tasks and send them to his workers. It is difficult for the manager to know whether his man finish the work or not. The manager had to call his man or talk with his man face to face to get the status of dispatched work.

The other two companies using spreadsheet managing their business focused on the management of job tickets. One reason was that tracking of job tickets were the core function for printers at present. Without the tracking of job tickets, printers couldn't handle customers' orders. Another reason was that it would be more complex if other information such as customer, material and cost were involved in the spreadsheets. With spreadsheets, the worker from schedule arranging department had to cut and paste tasks of working jobs from one file to another according to the status of the jobs. It was a tedious and error-prone task for department managers. Bosses of the two companies were interested in ERP system and expected ERP to help them improve their production effectively and efficiently. However, they wondered whether ERP system could be helpful as expected. They heard many stories about failed ERP project in printing industry. Most of the private printer preferred buying printing related devices to investing on information management system. There should be a solution to help printer owners solve their problems faced with low costs.

\section{Cloud Computing}

There are many different definitions about cloud computing. In 2009, Buyya and his colleagues defined cloud computing as "Cloud is a parallel and distributed computing system consisting of a collection of inter-connected and virtualized computers that are dynamically provisioned and presented as one or more unified computing resources based on service-level agreements (SLA) established through negotiation between the service provider and consumers."[3] The National Institute of Standards and Technology (NIST) characterizes cloud computing as "Cloud computing is a model for enabling convenient, on-demand network access to a shared pool of configurable computing resources (e.g. networks, servers, storage, applications, and services) that can be rapidly provisioned and released with minimal management effort or service provider interaction. This cloud model promotes availability and is composed of five essential characteristics, three service models, and four deployment models."[4]

The NIST draft definition goes on to describe three main cloud services: Infrastructure as a Service (IaaS), Platform as a Service (PaaS) and Software as a Service (SaaS).

IaaS provides consumers with administrative, web-based access to fundamental computing resources such as processing power, storage and networks. With IaaS, service users manage the application, data, runtime, middleware, and even the OS. The main part with the virtualization and hardware are managed and maintained by cloud provider.
Platform as a Service provides consumers with a stable online environment where they can quickly create and deploy web applications using browser-based software development tools. With PaaS, service user only has to manage the applications and data, and the cloud service provider manages the rest of infrastructure. There is less work involved in creating an application using PaaS than the traditional approach, which involves procuring and managing one or more servers for development, testing and production, and installing and configuring server software.

Software as a Service provides complete business applications delivered over the web. With SaaS, service provider manages the data and platform. End users are not responsible for supporting the application or any of the components. End users are basically paying a provider for a service, usually on a subscription basis. SaaS is probably the most prevalent of the cloud services at present. There are some famous SaaS services such as Google Doc, and Email.

There are four deployment models with the definition of the National Institute of Standards and Technology (NIST), which are public cloud, private cloud, community cloud and hybrid cloud. In general, the deploy models of cloud computing are largely divided in two types, private cloud and public Cloud. Hybrid cloud or community cloud is combination of Private and Public cloud.

\section{Cloud Based Printing Management System}

Until now, we have found few cloud computing related applications in printing industry. Although software giants, such as Microsoft, Amazon, Google and Oracle have already provided cloud computing services, it is hard to find a cloud computing service provided for printers. Google declared the cloud print which is used to connect printer to web. Founder, one of the most famous service providers for printing press in China, also declared that they had cloud computing based solution for color management. All the existing solutions cannot meet with the requirement of printing management.

Software as a service model (SaaS) is one of the best service model for most printing companies. For most printers, especially for small and median scale, there usually is no full time IT employee. Large amount of fund for MIS is almost impossible. So Infrastructure as a Service model (IaaS) and Platform as a service model (Paas) is not suitable for them. If the MIS system for printing company is provided as a service with cloud computing technology, printers do not need to invest on hardware for deploying and running the MIS system. There is also no cost for maintaining the MIS system. The only cost is the annual fee for the SaaS service.

There are other benefits besides low cost and no additional employee requirement. With Cloud-based printing MIS system, employees both ordinary ones and managers can access the system from personal computer or portable tablet or other smart mobile phone. For example, online workers can access the system and report the status of their working through web browser installed on their smart mobile phone. While business employees can input their orders information 
into the system and get the work to be processed as soon as possible without waiting until they get back to the company. This can solve information latency problem faced by many printers.

To provide a SaaS mode service to printers, we can deploy and run the system with Iaas or Paas service to make sure the system is elastics and the computing and other resources can increase or decrease automatically. However, more work has to be carried out with IaaS service than with Paas service to deploy printing MIS system.

\section{Design of a Prototype}

We designed a printing management system for a private printer. To demonstrate the characteristics of cloud computing system, we focused on the core manufacturing process, which is most printers care about currently. The process starts when sale man inputs the sale's order into the system and ends when the content of the order has been packaged.

Business process model and notation [5] (BPMN) is adopted to model the manufacturing process.

The print manufacturing process is shown in Figure 1.

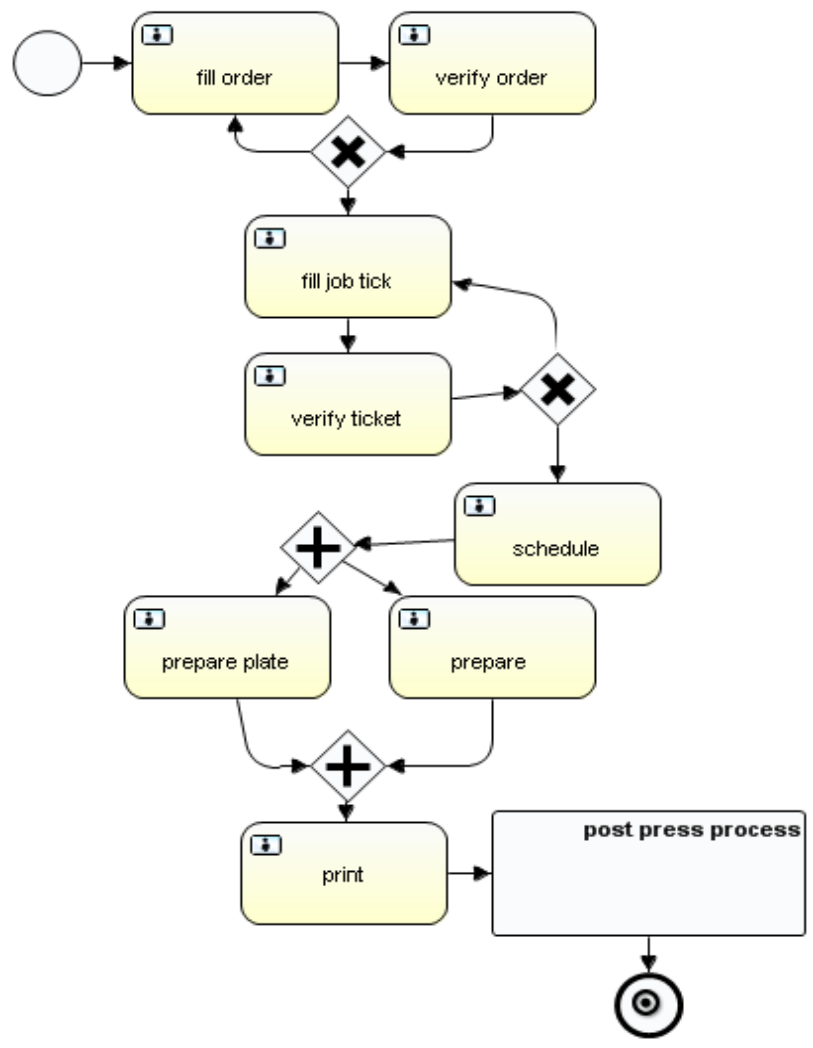

Fig. 1 Print manufacturing process

Sale man starts the process instance by filling an order form. The manager of sale department will verify the order and make a decision if the order is reasonable. If the answer is yes, then employee of job tickets management will generate a ticket. The manager of job tickets department will check the ticket. Once approved, the job ticket will be passed to schedule arranging department. After that plate and paper are prepared in parallel. When plate and paper are both ready, printing task starts. After printing task is finished, post press sub-process starts. There are many tasks within post press process, such as folding, gluing, binding, cutting and packing.

To simplify the management of underline cloud computing, we designed a prototype of printing manufacturing management and developed with Java EE technologies. Java Service Face framework [6] was adopted as web development framework. We deployed the prototype on the platform provided by CloudBees [7]. CloudBees is a cloud computing Platform as a Service provider, who provides limited computing and storage resources for free. Another advantage is that a Java web application can be deployed on CloudBees platform without changing except the configuration file. It was enough for us to demonstrate our designed system. In practice, more resource units can be bought according to customer's need without changing deployed application.

\section{Results}

After the print manufacturing management system has been deployed on CloudBees platform, users can access the application with web browser from anywhere at any time as long as internet connection is available. Employees from sale department can start the process instance by inputting a new order into the system. The manager of sale department is able to view all orders that need to be checked and approved in his or her working list. There are different views for different roles. Figure 2 shows the user interface for employees who are responsible for tasks arrangement.

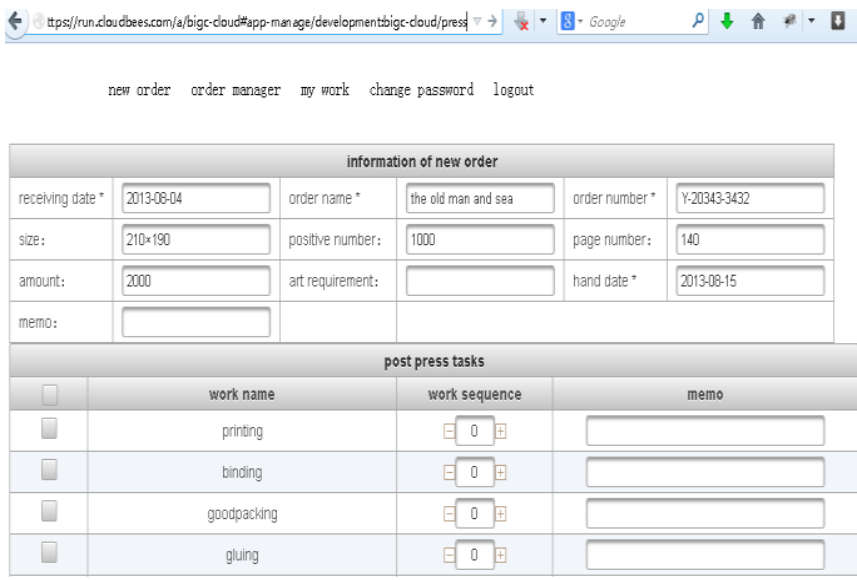

Fig. 2 New order input

Job ticket management has a different view which shows the status of orders being carried out as shown in Figure 3. For workers from prepress, press or post press department, after logged on the system, there is only information about tasks should be done. When a task is done, the worker can acknowledge the status of the task. Then the following tasks can start to be carried out. Figure 4 shows the user interface. 
Iy mork change password logout

\begin{tabular}{|c|c|c|c|c|c|c|}
\hline \multicolumn{7}{|c|}{ my tasks } \\
\hline order Number & size & requriement & memo & comment & finish flag & operation \\
\hline C programming & $210 \times 285$ & C programming & 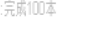 & & O no $O$ yes & ok \\
\hline Guidance for new students & $145 \times 210$ & Guidance for new students & 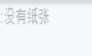 & & O no $O$ yes & ok \\
\hline The spring of HuluBeier & $147 \times 210$ & The spring of HuluBeier & & & 0 no $O$ yes & ok \\
\hline The beautity of logic & 正8K & The beautly of logic & & & O no $O$ yes & ok \\
\hline Red Cross & $170 \times 240$ & Red Cross & & & O no 0 yes & ok \\
\hline
\end{tabular}

Fig. 3 Task state acknowledge

executing orders departinent working finished orders departinent finished order order queny

\begin{tabular}{|c|c|c|c|c|c|}
\hline executing orders & \multicolumn{5}{|c|}{ order Hame : C programmining } \\
\hline Children's Tale & department & memo & start & firished & finished date \\
\hline Jata proogremmining & printing & 完感10本 & 0 & 8 & \\
\hline digest of Xinghus & suoxiqu & & 4 & 4 & \\
\hline 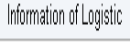 & macie & & 4 & 8 & \\
\hline Bible & paxilan & & 8 & 4 & \\
\hline Gills'stoly' & quecheynoin & & 8 & 8 & \\
\hline Cprogyeanminging & \multicolumn{5}{|c|}{\begin{tabular}{ll|l|}
44 & 4 & 1
\end{tabular}} \\
\hline
\end{tabular}

Fig. 4 Monitor states of being executed order

\section{Conclusion}

With cloud based print manufacturing information management system, users can access the application from anywhere at any time as long as internet connection is available. The application provides different view according to the roles of a user, which helps users focus on their own work. One limitation of this research is that the number of investigated printer is small. In the future, we should increase the number of samples. The other limitation is that this paper only represented a prototype of Saas application model. A practical system should be designed in the future. More aspects, including security should be considered in the future research.

\section{Acknowledgment}

Yongbin Zhang thanks Xiuli $\mathrm{Li}$ at RuiHe Printing Company for the helpful responses to printing order management questions and thanks Beijing Municipal Commission of Education for the financial support. Yongbin Zhang also thanks for the financial support provided by The Importation and Development of High-Caliber Talents Project of Beijing Municipal Institutions. Shulin Yang thanks Beijing Institute of Graphic Communication for the support.

\section{References}

[1] Dennis Mason. Print Business Management Software-How Does It Fit? GATFWorld, 2010, 2(1):27-28.

[2] Annual report of Chinese Printing press industry in 2011. DOI=http://info.printing.hc360.com/2012/01/091004429981.shtml.

[3] Rajkumar Buyya, James Broberg, and Andrzej Goscinski, Cloud Computing Principles and Paradigms, John Wiley \& Sons, Inc., Hoboken, New Jersey, 2010.

[4] The NIST Definition of Cloud Computing. DOI=http://csrc.nist.gov/publications/PubsSPs.html.

[5] Business Process Model and Notation (BPMN). http://www.omg.org/spec/BPMN/

[6] JSR-000314 JavaServer Faces 2.0.

DOI=http:// jcp.org/aboutJava/communityprocess/final/jsr314

[7] CloudBees platform. DOI=http://www.cloudbees.com/platform/platformoverview.cb 